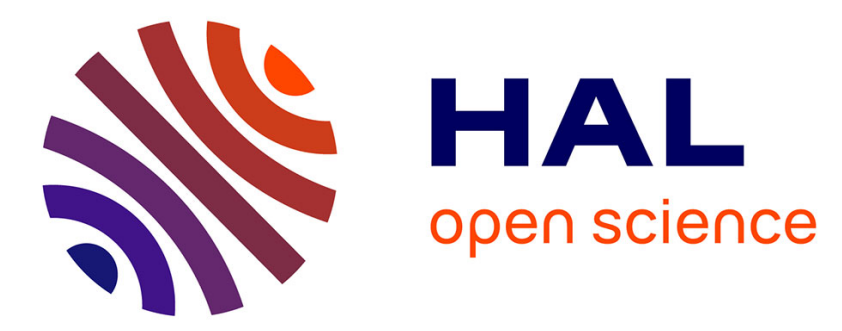

\title{
Analytic laws for direct calibration of discrete element modeling of brittle elastic media using cohesive beam model
}

\author{
Truong-Thi Nguyen, Damien André, Marc Huger
}

\section{- To cite this version:}

Truong-Thi Nguyen, Damien André, Marc Huger. Analytic laws for direct calibration of discrete element modeling of brittle elastic media using cohesive beam model. Computational Particle Mechanics, 2019, 6 (3), pp.393-409. 10.1007/s40571-018-00221-0 . hal-02304666

\section{HAL Id: hal-02304666 \\ https://hal.science/hal-02304666}

Submitted on 3 Oct 2019

HAL is a multi-disciplinary open access archive for the deposit and dissemination of scientific research documents, whether they are published or not. The documents may come from teaching and research institutions in France or abroad, or from public or private research centers.
L'archive ouverte pluridisciplinaire HAL, est destinée au dépôt et à la diffusion de documents scientifiques de niveau recherche, publiés ou non, émanant des établissements d'enseignement et de recherche français ou étrangers, des laboratoires publics ou privés. 


\title{
Analytic laws for direct calibration of discrete element modeling of brittle elastic media using cohesive beam model
}

\author{
Truong-Thi Nguyen · Damien André · Marc Huger
}

Received: date / Accepted: date

\begin{abstract}
In this study, a new methodology for the calibration of microscopic parameters for the Cohesive Beam Model (CBM) of discrete element method (DEM) applied to elastic brittle material is presented. This method enables the entry of material mechanical values directly into DEM simulations without any calibration steps. Several DEM simulations of tensile tests with different microscopic parameter values were carried out to generate a database of macroscopic parameter responses. This database was analyzed in order to deduce analytic laws by using non linear least square method. To validate the proposed calibration method, DEM simulations, that use the results of the calibrated microscopic parameters, were carried out. The macroscopic responses were compared to theoretical or experimental values. These validation tests were performed separately for two typical brittle elastic materials, i.e., soda-lime glass and alumina, with different shapes/sizes of discrete domain and various boundary conditions. Results between numerical and experimental values are in good accordance regarding the variability induced by this stochastic approach.
\end{abstract}

Keywords Discrete Element Method (DEM) - Brittle materials · Cohesive beam bond . Calibration $\cdot$ Analytical law between microscopic /macroscopic parameters

\section{Introduction}

In the mechanical engineering field, continuum approaches are widely used to model continuous media through reliable stress-strain laws. However, continuum approaches can not manage easily fracture phenomena that involve high amount of discontinuities, complex

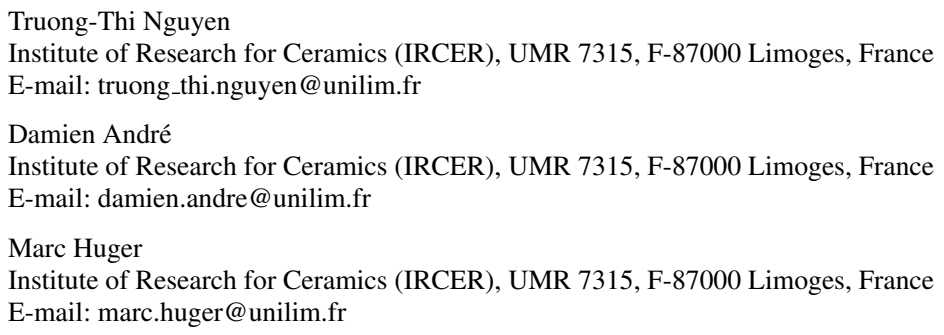


crack geometries and crack closures. Discrete Element Method (DEM) could be an alternative way to study these phenomena because it takes naturally into account discontinuities. The DEM implements a group of distinct elements (also named discrete elements) in interaction through contacts or cohesive laws. The advantages of this method are the description, in a natural way, of crack initiations, crack propagations, their coalescences and closures. However, the fundamental difficulty of this approach is to simulate quantitatively the continuum behavior [35].

Different contact models of DEM can be found in the literature. For example, in the bonded-particle method, developed by Potyondy et al [35] for modeling rocks, the material is modeled as assembly of nonuniform-sized rigid spherical particles that may be bonded together at their contact points. Mainly, two models of bonds are used in bonded-particle method: contact bond and parallel bond. The contact bond model can be considered as a pair of elastic springs with constant normal and shear stiffnesses that act at contact points between particles. These two springs have specified tensile and shear strength which limit the tensile force and shear force respectively. The bond is broken if the normal force or shear force exceeds the corresponding bond strength. This model allows only transmission of force through contact. The parallel bond model can be consider as a set of elastic springs with constant normal and shear stiffnesses, uniformly distributed over a cross-section lying on the contact plane and centered at the contact point. Parallel bond can transmit both force and moment through contact. Force and moment act on the two contacting particles and can be related to maximum normal and shear stresses. If one of these maximum stresses exceeds its corresponding bond strength, the parallel bond is broken. The bonded-particle method is widely used to study fracturing and fragmentation processes of brittle materials. This method is implemented in many common DEM softwares, such as PFC [22] and YADE [25]. However, one of major drawbacks of the bonded-particle methods is that determining the proper set of bond properties (microscopic parameters) is difficult. In fact, the input parameters of the model are set at the microscopic scale and they do not correspond to the material properties. A pre-processing step is necessary (calibration process) to reach quantitative results. This drawback is due to the high number of microscopic parameters, for example, 10 microscopic parameters are needed to determine the contact bond model [47]: contact modulus, stiffness ratio, friction coefficient, contact-bond normal strength, contactbond shear strength, etc. In parallel, the Cohesive Beam Model (CBM) has been developed by different authors $[1,3,6,15,29]$. This contact model is able to simulate the behaviour of perfect brittle-elastic media defined by a Young's modulus, a Poisson's ratio and a tensile failure strength. In this framework, discrete elements are connected by cylindrical cohesive beams which are able to work in tension, compression, bending and torsion. The EulerBernoulli beam theory is implemented to compute the beam forces and moments. In the contact model proposed by André et al [1,3], the cohesive beam bond is defined by only two microscopic parameters. The calibration process of this model seems to be less complex than the previous bond models.

As mentioned previously, the first step of quantitative simulations with DEM, consists in calibrating the microscopic parameters (bond properties) at the discrete element scale. Typically, calibration is achieved through a trial-and-error procedure in which microscopic parameters are adjusted until the DEM results match the expected behaviour. Such calibration step is necessary to achive quantitative results but it is complicated and time consuming. Many calibration methodologies has been reported. Dimensionless relationships between DEM micro parameters and macroscopic properties were established in different researches $[12,36,49]$. In these works the micromechanical parameters have been determined using the methodology developed by Huang [19], based on the combination of the 
dimensional analysis with numerical simulation of the standard laboratory tests for rocks. Rojek et al [36] introduce a dimensionless micro-to-macro relationships for 2D and 3D discrete element models by analyzing macroscopic responses of Brazilian tests and Unconfined Compression Tests (UCT). In this research, the authors consider the parallel bond model which is determined by 6 microscopic parameters. The considered macroscopic rock properties are the Young's modulus $E$, Poisson's coefficient $v$, compressive strength $\sigma_{c}$ and tensile strength $\sigma_{t}$. Based on Buckingham $\pi$ theorem [27], 4 dimensionless functional relationships linking macroscopic and microscopic parameters were established. Simulations of both the UCT and Brazilian tests have been performed with different values of stiffness ratio in order to provide specific forms of dimensionless relationships. The proposed relationships were applied for modelling rock cutting and seems to give satisfactory results. However, assumption about normal and shear cohesive bond strength ratio is required and no analytic function is proposed by authors to describe micro-to-macro relationships. Recently, several calibration methods able to describe evolution of macroscopic parameters versus microscopic parameters have been developed without any assumption [10,16]. Estay et al [10] propose a matrix methodology to predict the Young's modulus of a specimen as a function of bond elastic parameters. This calibration method is validated through uniaxial compression tests. Han et al [16] propose a fitting function to characterise relationship between macroscopic tensile strength and the micro mechanical breakage parameters by using simulations of Brazilian test. In these researches, the calibration method is applied for only one macroscopic parameter and validated through only one type of numerical experiment. The precision of the proposed calibration methods are not discussed for other type of DEM simulations.

The present study focuses on the Cohesive Beam Model proposed by André et al $[1,3]$. Relationships between microscopic and macroscopic parameters of Cohesive Beam Model are deduced from 8,000 uniaxial virtual tensile tests. In order to deduce macro-to-micro analytic laws, this high amount of data is analyzed through non linear least square methods. Then, the deduced relationships are verified by using academic test cases : tensile test, hydrostatic compression test, torsion test and brazilian test. This methodology considers three mechanical properties that define a perfect elastic brittle behaviour: the Young's modulus, the Poisson's ratio and the tensile strength. The goal of this work is to propose a fast and robust DEM calibration method that allows direct computation of microscopic parameter values from material properties. This method could eliminate the need for repeating trialand-error calibration and facilitate the usage of DEM for non-specialists. In addition, several improvements are proposed for the calculation of equivalent Cauchy stress tensors involved in the local failure criterion computation.

Following this introduction, the present paper is divided into 4 sections. Section 2 introduces the DEM model applied to brittle elastic media. In this section, a virial stress-based model to simulate brittle behaviour is presented. In section 3, a direct calibration method of microscopic parameter of DEM is proposed. In section 4, the proposed method is validated through numerical experiments that involve several virtual sample shapes. Convergent results of macroscopic properties are obtained with the difference to theoritical values lower than $8 \%$. In addition, the fracture criterion is validated through glass and alumina disk subjected to Brazilian tests, torsion tests and compressive tests. Finally, this paper will be closed by several conclusions and perspectives. 


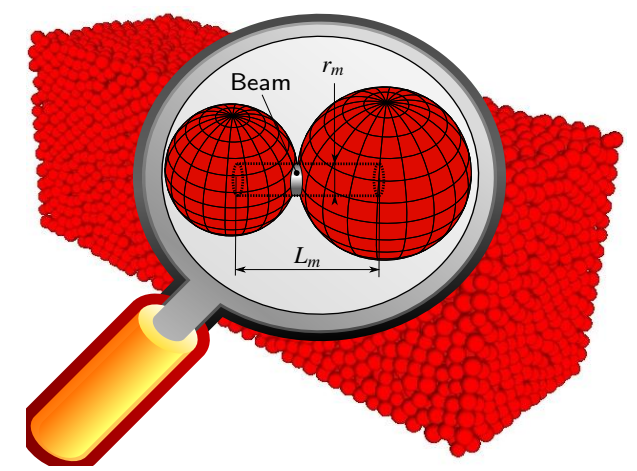

Fig. 1 The cohesive beam bond

\section{Discrete element model applied to continuous media}

In the present paper, the variation of DEMs described by André et al. is used to model continuous materials $[1,4,23]$. This approach is "hybrid" between lattice models and particle models as it was first proposed by Potyondy [35]. The simulations presented in this paper were performed with the GranOO software [3]. GranOO uses an explicit integration scheme, named Verlet velocity scheme, that is well suited to high velocity phenomena, such as crack propagation or impact and massive DEM simulations [37]. At this time, GranOO embeds some models that enable the quantitative simulation of mechanical, thermal and electrical behaviour of continua with DEM $[3,20,45]$. This section introduces the main aspects of the numerical models to model mechanical behaviour of continuous media.

\subsection{Cohesive beam bond model}

The cohesive beam model was first introduced by H. J. Herrmann in 1988 [17]. This model was first used in a 2D ordered lattice network [40,41], and later, in disordered 2D lattice networks [7, 8, 21, 26, 39]. In reference [39], the authors have considered that microscopic and macroscopic Young's modulii and Poisson's ratios are similar. The related calibration method is not described in depth. Authors recommend trial and error tests using experimental and numerical approaches. In the present study, mechanical properties of the cohesive beams will not be considered as similar to the reference material. So, microscopic local properties could be driven to produce the desired behaviors at the macroscopic scale.

Fig. 1 draws two discrete elements bonded by a cohesive beam. The cylindrical geometry is chosen because it's dimensional description requires only two independent parameters: a length $L_{m}$ and a radius $r_{m}{ }^{1}$. In addition, mechanical properties of cohesive beams are: a Young's modulus $E_{m}$ and a Poisson's ratio $v_{m}$. These four geometric and mechanical parameters fully describe a cohesive beam. Cohesive beams are mass-less; mass properties are assigned only to discrete elements.

For the sake of clarity Fig. 2 shows a configuration in which the discrete elements have been moved away. The cohesive beam is symbolized by its median line. Both cohesive

\footnotetext{
1 To distinguish micro from macro properties, micro parameters are denoted by ' $m$ ' indices and macro parameters by ' $M$ ' indices.
} 
bond ends are fixed to the discrete element centers $O_{1}$ and $O_{2}$. The discrete element frames $F_{1}\left(O_{1}, \mathbf{X}_{\mathbf{1}}, \mathbf{Y}_{\mathbf{1}}, \mathbf{Z}_{\mathbf{1}}\right)$ and $F_{2}\left(O_{2}, \mathbf{X}_{\mathbf{2}}, \mathbf{Y}_{\mathbf{2}}, \mathbf{Z}_{\mathbf{2}}\right)$ are oriented such that $\mathbf{X}_{\mathbf{1}}$ and $\mathbf{X}_{\mathbf{2}}$ are normal to the beam cross section ends. At the initial time, the beams are relaxed (figure 2(a)). Fig. 2(b) shows the cohesive beam in a arbitrary loading state.

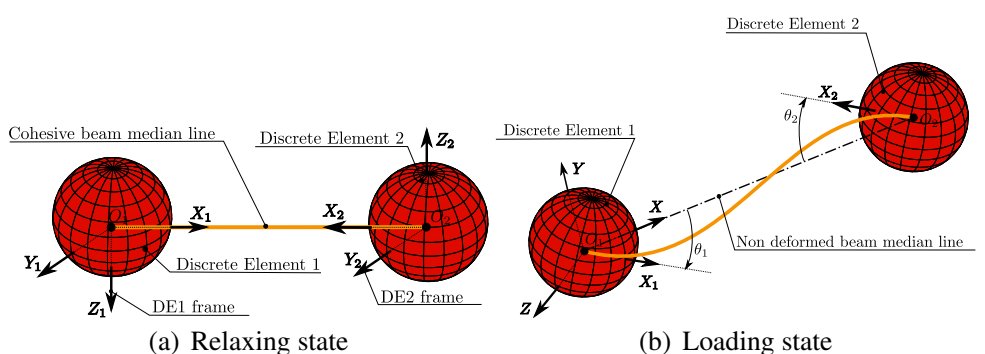

Fig. 2 Cohesive beam bond configurations

The analytic model of Euler-Bernoulli beam is well known [46]. Indeed, special attention are given to local frame positionning. Figure 2(b) illustrates the beam local frame positioning. The center of discrete element $1\left(O_{1}\right)$ is considered as the origin. The "aligned" configuration, in which $\mathbf{O}_{1} \mathbf{O}_{2}=k \mathbf{X}_{1}=-k \mathbf{X}_{2}$, is considered as the non-bending state and is taken as reference. Consequently, the cohesive beam local frame $F(O, \mathbf{X}, \mathbf{Y}, \mathbf{Z})$ is oriented such that (see figure 2(b)):

$$
\mathbf{X}=\frac{\mathbf{O}_{1} \mathbf{O}_{2}}{\left\|\mathbf{O}_{1} \mathbf{O}_{2}\right\|} \text { and } \mathbf{Y}=\mathbf{X} \wedge \mathbf{X}_{\mathbf{1}} \text { and } \mathbf{Z}=\mathbf{X} \wedge \mathbf{Y}
$$

In the local frame $F$, the deflections at $O_{1}$ and $O_{2}$ are null. Cross section bending rotations at $O_{1}$ and $O_{2}$ are defined, respectively, by $\theta_{1}=\left(\mathbf{X}, \mathbf{X}_{1}\right)$ and $\theta_{2}=(-\mathbf{X}, \mathbf{X} \mathbf{2})$. In addition, the small rotation hypothesis is used at local frame $\left(\theta_{1}\right.$ and $\left.\theta_{2}<12^{\circ}\right)$. Consequently, the force and torque reactions acting on discrete elements 1 and 2 are:

$$
\begin{aligned}
& \mathbf{F}_{\mathbf{D E} 1}=+E_{m} S_{m} \frac{\Delta l_{m}}{l_{m}} \mathbf{X}-\frac{6 E_{m} I_{m}}{l_{m}^{2}}\left(\left(\theta_{2 z}+\theta_{1 z}\right) \mathbf{Y}+\left(\theta_{2 y}+\theta_{1 y}\right) \mathbf{Z}\right) \\
& \mathbf{F}_{\mathbf{D E} 2}=-E_{m} S_{m} \frac{\Delta l_{m}}{l_{m}} \mathbf{X}+\frac{6 E_{m} I_{m}}{l_{m}^{2}}\left(\left(\theta_{2 z}+\theta_{1 z}\right) \mathbf{Y}-\left(\theta_{2 y}+\theta_{1 y}\right) \mathbf{Z}\right) \\
& \mathbf{T}_{\mathbf{D E 1}}=+\frac{G_{m} I o_{m}}{l_{m}}\left(\theta_{2 x}-\theta_{1 x}\right) \mathbf{X}-\frac{2 E_{m} I_{m}}{l_{m}}\left(\left(\theta_{2 y}+2 \theta_{1 y}\right) \mathbf{Y}-\left(\theta_{2 z}+2 \theta_{1 z}\right) \mathbf{Z}\right) \\
& \mathbf{T}_{\mathbf{D E 2}}=-\frac{G_{m} I o_{m}}{l_{m}}\left(\theta_{2 x}-\theta_{1 x}\right) \mathbf{X}-\frac{2 E_{m} I_{m}}{l_{m}}\left(\left(2 \theta_{2 y}+\theta_{1 y}\right) \mathbf{Y}-\left(2 \theta_{2 z}+\theta_{1 z}\right) \mathbf{Z}\right)
\end{aligned}
$$

where:

- $\mathbf{F}_{\mathrm{DE} 1}$ and $\mathbf{F}_{\mathrm{DE} 2}$ are the beam force reactions acting on discrete elements 1 and 2.

- $\mathbf{T}_{\mathbf{D E} 1}$ and $\mathbf{T}_{\mathbf{D E} 2}$ are the beam torque reactions acting on discrete elements 1 and 2. 
- $l_{m}$ and $\Delta l_{m}$ are the initial beam length and the longitudinal extension.

- $\theta_{\mathbf{D E 1}}\left(\theta_{1 x}, \theta_{1 y}, \theta_{1 z}\right)$ and $\theta_{\mathbf{D E 1}}\left(\theta_{2 x}, \theta_{2 y}, \theta_{2 z}\right)$ are the rotations of beam cross section at the points $O_{1}$ and $O_{2}$ expressed in the beam local frame.

- $S_{m}, I o_{m}$ and $I_{m}$ are the beam cross section area, polar moment of inertia and moment of inertia along $\mathbf{Y}$ and $\mathbf{Z}$.

- $E_{m}$ and $G_{m}$ are the Young's and shear modulus.

Reaction force and torque are expressed in the beam local frame $F(O, \mathbf{X}, \mathbf{Y}, \mathbf{Z})$. With such model, the cohesive beam bond is defined by four parameters:

- two geometrical parameters: length $L_{m}$ and radius $r_{m}$.

- two mechanical parameters: Young's modulus $E_{m}$ and Poisson's ratio $v_{m}$.

Indeed, the cohesive beam length value $L_{m}$ depends on the distance between discrete element centers and is not a free parameter because it is defined through the compact process (see section 2.3). In addition, the $v_{m}$ has no influence on the macroscopic elastic behavior [1]. So, only two parameters $E_{m}$ and $r_{m}$ must be calibrated to match macroscopically a perfect elastic media defined by a Young's modulus $E_{M}$ and a Poisson's ratio $v_{M}$.

The dimensionless beam radius noted $r_{m}$ is preferred to the beam radius. This dimensionless beam radius is defined as the ratio between the cohesive beam radius and the average discrete element radius. The $r_{m}$ value is the same for all the cohesive beams involved in a discrete model. It allows us to consider the cohesive beam as a length-free model. It means that the input values, i.e, the Young's modulus $E_{m}$ and the radius ratio $r_{m}$, do not depend on the length of the sample. In other words, the mechanical elastic property of a discrete sample, defined by its apparent Young's modulus and Poisson's ratio, do not depend on its size. This is an important feature of the cohesive beam model.

\subsection{Failure criterion through virial stress tensor}

Within the discrete element approach, cracks are generally simulated by breaking cohesive bonds if an imposed criterion is reached [44] [42]. The main existing approaches are based on the computation of bond strains [6] or stresses [35]. However, these approaches seems to be not suitable for simulating complex crack paths such as the Hertzian cone crack exhibited by fused silica glass [4]. In this study, a fracture criterion, based on the computation of an equivalent Cauchy stress tensor (the so-called virial stress tensor) and maximal principal stress value, is involved. Several researches pointed out that virial stress-based model seems to be suitable for simulating elastic brittle material [4, 14,24]. Mainly, virial stress tensors are used as post-processing tool for computing stress maps in a DEM calculation. Here, this mathematical tool is used during a DEM calculation for computing accurately the failure criterion in terms of maximal stresses. Because previous formulations lack of accuracy, a new formulation is proposed here by considering several neighbour level as explained later. The equivalent Cauchy stress tensor $\overline{\bar{\sigma}}_{\Omega}$ is computed as [51]:

$$
\overline{\bar{\sigma}}_{\Omega}=\frac{1}{2 \Omega} \sum_{i \in \Omega}\left(\frac{1}{2} \sum_{j=1}^{N}\left(\mathbf{r}_{i j} \otimes \mathbf{f}_{i j}+\mathbf{f}_{i j} \otimes \mathbf{r}_{i j}\right)\right)
$$

where :

- $\Omega$ is the volume associated to the virial stress computation,

- $\overline{\bar{\sigma}}_{\Omega}$ is the equivalent Cauchy stress tensor of the considered volume $\Omega$, 
- $j$ takes values 1 to $N$ neighbors of the discrete element $i$,

- $\otimes$ is the tensor product between two vectors,

- $\mathbf{f}_{i j}$ is the force imposed on the discrete element $i$ by a cohesive beam that bonds the discrete element $i$ to its "neighbour discrete element" $j$,

- $\mathbf{r}_{i j}$ is the relative position vector between the center of the two bonded discrete elements $i$ and $j$.

As illustrated on Fig. 3, the stress tensor is always computed for the central discrete element. Thus, different volumes $\Omega$ can be considered depending on the neighbor level. Neighbor elements that connect directly with the central element through cohesive beams are defined as neighbor level 0 . Discrete elements that connect directly with the discrete elements in neighbor level 0 are defined as neighbor level 1 and so on. The volume $\Omega$ is simply defined as :

$$
\Omega=\frac{1}{f_{v}} \sum_{i \in \Omega} \Omega_{i}
$$

where :

- $i$ is related to a discrete element,

- $\Omega_{i}$ is the volume of the discrete element $i$ and

- $f_{v}$ is the global volume fraction of the discrete domain.

With such a model, high value of neighbour level corresponds to non-local and averaged stress tensor while low value of neighbour level is related to local value with lower precision. A good compromise of the neighbour level is discussed further in this section.

To model brittle failure, the fracture criterion assumes that fracture occurs if the maximal principal stress $\sigma_{I}$, deduced from the virial stress, is higher than a threshold value $\sigma_{m}$ :

$$
\sigma_{I} \geq \sigma_{m}
$$

where $\sigma_{m}$ is the microscopic fracture threshold. The maximal principal stress $\sigma_{I}$ is deduced from the virial stress $\overline{\bar{\sigma}}_{\Omega}$ thanks to linear algebra computations [43] able to compute eigen vector $\left(\sigma_{X X}, \sigma_{Y Y}, \sigma_{Z Z}\right)$ from symmetric matrices. In addition, the related principal base $\mathscr{F}(\mathbf{X}, \mathbf{Y}, \mathbf{Z})$, in which the virial stress tensor is diagonal, is computed. The maximal principal stress is simply deduced as :

$$
\sigma_{I}=\max \left(\sigma_{X X}, \sigma_{Y Y}, \sigma_{Z Z}\right)
$$

In a previous study [4], all the bonds that belong to a discrete element that reaches the fracture criterion were broken. As a consequence, a debonded discrete element occurs and a debris is created. This can lead to a loss of mass and volume of the virtual sample. To avoid this problem, when failure occurs, only one cohesive beam is broken. Hence, a computation to select the broken beam is required. The failure direction $\mathbf{d}_{i}$ of discrete element $i$ is assumed to be the direction of the eigen vector of virial stress tensor that corresponds to the maximal principal stress $\sigma_{I}$. This direction can be simply deduced from the $\mathscr{F}(\mathbf{X}, \mathbf{Y}, \mathbf{Z})$ frame where $\mathbf{d}_{i}$ corresponds to the $\mathbf{X}, \mathbf{Y}$ or $\mathbf{Z}$ axes (see equation 8). The cohesive beam included in the neighbour level 0 which has the most similar direction with $\mathbf{d}_{i}$ is broken.

The accuracy of different neighbor levels are examined in order to choose the most appropriate one. Several simulations of tensile tests (see section 2.4) with neighbor levels 0,1 and 2 were carried out. The value of microscopic Young's modulus $E_{m}$ was arbitrary fixed at $500 \mathrm{GPa}$. Radius ratio was set from 0.2 to 1.0. The macroscopic normal stress $\sigma_{M_{x x}}$ 


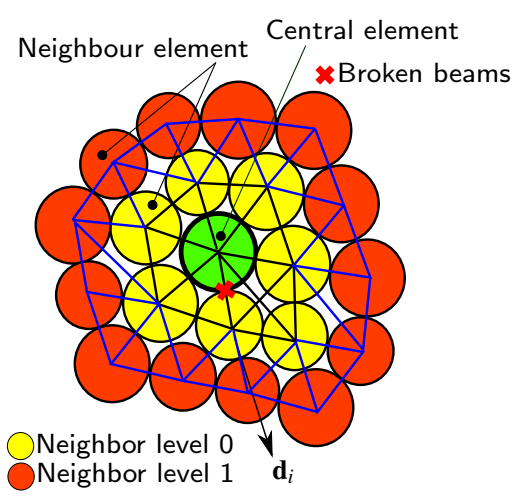

Fig. 3 Illustration of neighbour levels in 2D used for virial stress computation

is computed thanks to Equation 10 (see next section). This value is compared to the mean $<\sigma_{m_{x x}}>$ of the $x x$ component of local virial stress tensor $\sigma_{m_{x x}}$. The discrete elements in the loaded boundaries are not taken into account. Finally, the relative difference between $<\sigma_{m_{x x}}>$ and $\sigma_{M_{x x}}$ is computed by the following equation.

$$
\text { error }=\frac{<\sigma_{m_{x x}}>-\sigma_{M_{x x}}}{\sigma_{M_{x x}}} \times 100
$$

The results are summarized in Table 1. This table shows that simulations with neighbor level 1 have the lowest difference (lower than $2 \%$ ). This results comes from the case of coordination number $c n=10$. The same tendency was observed for other coordination number. Consequently, the neighbour level equal to 1 will be used to perform DEM simulations in the presented study.

Table 1 Average relative errors between local virial stress and global normal stress

\begin{tabular}{llll}
\hline$r_{m}$ & lv 0 & lv 1 & lv 2 \\
\hline 0.2 & $1.48 \%$ & $0.66 \%$ & $1.37 \%$ \\
0.4 & $1.28 \%$ & $1.25 \%$ & $1.95 \%$ \\
0.6 & $1.44 \%$ & $1.38 \%$ & $2.05 \%$ \\
0.8 & $1.59 \%$ & $1.42 \%$ & $2.08 \%$ \\
1.0 & $1.62 \%$ & $1.51 \%$ & $2.14 \%$ \\
\hline
\end{tabular}

\subsection{Geometrical properties of discrete domain}

In this study, continuum is represented by a discrete domain which is an assembly of non deformable spherical discrete elements. In such type of discrete domain, voids exist between elements. To reduce these undesirable voids, a compaction process is accomplished by an original algorithm, named cooker, detailed and explained in [23]. This algorithm ensures that discrete domains satisfy a good level of contact isotropy. The contact isotropy is defined here using an original statistical tool that involves 3D histograms, solid angle and platonic solids. More information about this intuitive 3D statistical tool are given in [1]. 
To guarantee a good level of contact isotropy, discrete element radii are randomly choosen through a uniform distribution with a range equal to $25 \%$ [1]. This randomization process prevents ordered configuration, also known as crystallization, in the obtained packing [33]. The cooker algorithm is able to fill a space of arbitrary shape with spherical discrete elements. It stops when the coordination number reach the 6.2 value that corresponds to the definition of a random close packing [13]. Then, a post processing algorithm is involved for replacing all the contacts by cohesive beams. This step may include a dichotomy algorithm ables to fit a given value of coordination number. The discrete element radii artificially expanses until it reaches the expected coordination number value. This algorithm allows us to choose the coordination number value and to connect elements even if they are not really in contact. As it was shown in [50] structural properties of assemblies has strong effects on apparent elastic responses. So, this post-processing step, able to fit a coordination number, enables to study the influence of the coordination number the macroscopic Young's modulus and Poisson's ratio.

\subsection{Tensile test simulation and computational methods of elastic reponses}

In discrete element models, macroscopic parameters can not be introduced directly as input parameters. Indeed, they need to be measured numerically through virtual experiments. Here, uniaxial tensile test simulations are used for deducing the apparent Young's modulus, Poisson's ratio and failure strength. For such simulations, cubic samples are build with the cooker algorithm (see previous section) with length of 2 millimeters and contains around 10,000 discrete elements. As it was shown in $[1,2,4]$, this number of discrete elements ensures a good level of convergence. It means that if higher values of element are choosen, the obtained results will not be different from those one obtained with 10,000 elements. In order to load the sample, opposite displacements at constant velocity are imposed to the discrete elements that belong to the $S_{x+}$ and $S_{x-}$ faces of these cubic domains (Fig. 4).

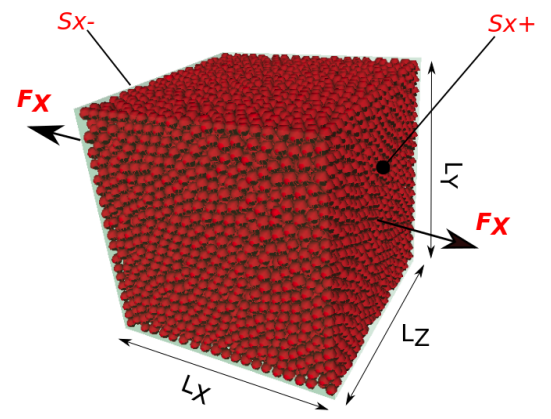

Fig. 4 The cubic discrete domain used to perform tensile tests simulation

The lengths $L_{x}, L_{y}$ and $L_{z}$ of the bounding box associated to the discrete domains are updated during the computation. This is done by taking into account the mean position of the discrete elements that belong to the box faces. Finally, these bounding length $L_{x}, L_{y}$ and $L_{z}$ are computed by adding the mean diameter of the discrete elements. From the knowledge of the bounding box lengths $L_{x}, L_{y}$ and $L_{z}$ and the applied force $F_{x}$ (Fig. 4), the average macroscopic normal stress $\sigma_{M x x}$ along the $\mathrm{x}$ axis can be deduced as: 


$$
\sigma_{M x x}=\frac{F_{x}}{L_{y} \times L_{z}}
$$

The macroscopic engineering strain $\varepsilon_{M x x}, \varepsilon_{M y y}$ and $\varepsilon_{M z z}$ along the $\mathrm{x}, \mathrm{y}$ and $\mathrm{z}$ axes are expressed as :

$$
\varepsilon_{M x x}=\frac{\Delta L_{x}}{L_{x}} \quad \varepsilon_{M y y}=\frac{\Delta L_{y}}{L_{y}} \quad \varepsilon_{M z z}=\frac{\Delta L_{z}}{L_{z}}
$$

So, the macroscopic Young's modulus $E_{M}$ is :

$$
E_{M}=\frac{\sigma_{M x x}}{\varepsilon_{M x x}}
$$

The macroscopic Poisson's ratio $v_{M}$ is computed by averaging the macroscopic Poisson's ratio values along the $\mathrm{y}$ and $\mathrm{z}$ axes as :

$$
\begin{gathered}
v_{M y}=-\frac{\varepsilon_{M y y}}{\varepsilon_{M x x}}, v_{M z}=-\frac{\varepsilon_{M z z}}{\varepsilon_{M x x}} \\
v_{M}=\frac{1}{2}\left(v_{M y}+v_{M z}\right)
\end{gathered}
$$

where $v_{M y} \approx v_{M y}$. Finally, the macroscopic failure stress $\sigma_{M}$ is the macroscopic tensile stress $\sigma_{M x x}$ when the failure occurs.

The density of discrete elements are chosen in order to fit a continuum as :

$$
\rho_{m}=\frac{\rho_{M}}{f_{v}}
$$

where $f_{v}$ is the volume fraction of the assembly (mainly equal to 63\%), $\rho_{M}$ is the density of the simulated material and $\rho_{m}$ is the density affected to the discrete elements. So, the mass $m_{i}$ of a discrete element $i$ is computed as :

$$
m_{i}=\rho_{m} \times V_{i}
$$

where $V_{i}$ is the volume of a discrete element $i$.

\subsection{Overview of the numerical scheme}

The temporal differential equation is solving by using an explicit integration scheme that is well suited for massive DEM simulations [37] and high velocity phenomena, such as crack propagation or impact. The velocity Verlet scheme is chosen for its simplicity and efficiency. The discrete element orientations are described by quaternions, which provide an efficient way of computing the discrete element rotations [34]. The quaternions must be normalized at every time step to prevent numerical drift. If it is required, a pure numerical damping factor is introduced in the numerical scheme as described by Tchamwa and Wielgosz [30] to allow a quick convergence rate toward the static solution. 


\section{Direct calibration method of microscopic parameters}

The first step of a quantitative simulation with DEM, consists in calibrating microscopic parameters of the discrete element model. According to Marigo et al. [31], this calibration step is the major obstacle on the use of DEM in industry and applied engineering sciences. The purpose of this section is to find analytic relationships between microscopic parameters, at the discrete element scale $\left(r_{m}, E_{m}, \sigma_{m}\right)$, and the macroscopic properties, at the scale of numerical sample $\left(v_{M}, E_{M}, \sigma_{M}\right)$ by considering structural effect such as the coordination number $c_{n}$ in order to skip trial-and-error and time consuming calibrations.

\subsection{Calibration of microscopic elastic parameters}

In this section, three relations are studied:

1. According to the parametric study of Andre et al., macroscopic Poisson's ratio $v_{M}$ is independent from microscopic Young's modulus $E_{m}$. Therefore, the evolution of the macroscopic Poisson's ratio $v_{M}$ versus radius ratio $r_{m}$ is studied : $v_{M}=f_{1}\left(r_{m}\right)$

2. Relation between macroscopic Young's modulus $E_{M}$ and microscopic Young's modulus $E_{m}$ and radius ratio $r_{m}: E_{M}=f_{2}\left(E_{m}, r_{m}\right)$

3. Relation between the macroscopic parameters $\left(E_{M}, v_{M}\right)$ and coordination number $c n$

Series of uniaxial tensile test simulations with different values of microscopic parameters (see Table 2) are carried out. The total number of simulations is $4 \times 5 \times 5 \times 16=1,600$ for this parametric study. These numerical tensile tests were presented in the section 2.4. Each set of microscopic and structural parameters $\left(r_{m}, E_{m}, c n\right)$ gives a set of macroscopic parameters $\left(v_{M}, E_{M}\right)$. Based on the obtained data of these tests, non-linear least squares methods $[28,32]$ are applied in order to find out the fitting functions that best describe the considered relations.

Table 2 Value of microscopic parameters

\begin{tabular}{ccc}
\hline & Values & Tot. number \\
\hline Sample & 1234 & 4 \\
$E_{m}(\mathrm{GPa})$ & 5001000150020002500 & 5 \\
$r_{m}(-)$ & 0.20 .40 .60 .81 .0 & 5 \\
$c n(-)$ & 5.566 .577 .588 .599 .51010 .51111 .512 .513 & 16 \\
\hline
\end{tabular}

There are probably many functions which can describes these macro-to-micro relationships. Indeed, the chosen fitting function must satisfy the following criteria:

1. the coefficient of determination must be in range: $R^{2} \in[0.98 ; 1]$,

2. the difference between the fitted curves and the data scatter must be lower than $1 \%$,

3 . the evolution of fitting function coefficients versus coordination number must be describable by a fitting function,

4. if many functions satisfy the three previous criteria, the function that involves the lowest number of coefficients is chosen.

After examining several fitting functions, one find that the relations $v_{M}=f_{1}\left(r_{m}\right)$ and $E_{M}=$ $f_{2}\left(E_{m}, r_{m}\right)$ could be described with the following fitting functions :

$v_{M}=f_{1}\left(r_{m}\right)=a_{1}+b_{1} \cdot r_{m}+c_{1} \cdot r_{m}^{2}+d_{1} \cdot r_{m}^{3}$ 
$E_{M}=f_{2}\left(E_{m}, r_{m}\right)=E_{m} \cdot\left(a_{2}+b_{2} \cdot r_{m}+c_{2} \cdot r_{m}^{2}+d_{2} \cdot r_{m}^{3}\right)$

In addition, the related fitted curves and surface are shown in Fig. 5(a) and 5(b).

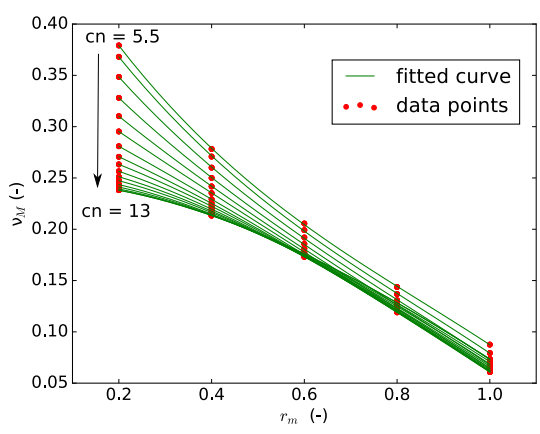

(a) Fitted curve $v_{M}=f_{1}\left(r_{m}\right)$ corresponding to all values of $\mathrm{cn}$

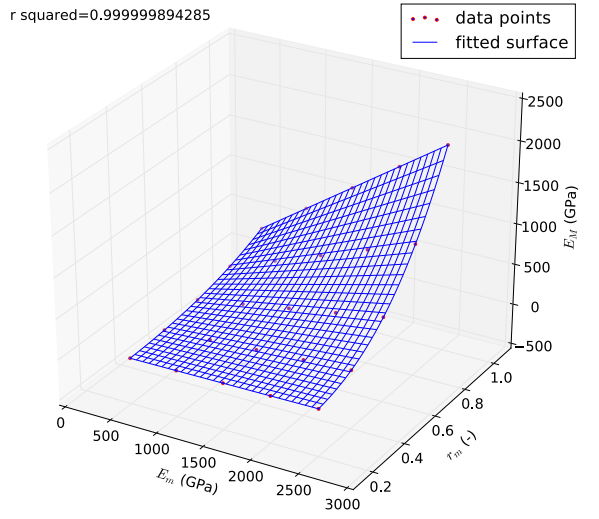

(b) Fitted surface $E_{M}=f_{2}\left(E_{m}, r_{m}\right)$, case of $\mathrm{cn}=6$

Fig. 5 Macroscopic Young's modulus and Poisson's ratio fitted curves and surface

In the fitted function related to equations 17 and 18, the coefficients $a_{1}, b_{1}, c_{1}, d_{1}$ and $a_{2}, b_{2}, c_{2}, d_{2}$ depend on coordination number. Again, the fitting functions that describe relationships between these considered coefficients and coordination number $c_{n}$ were found thanks to non-linear least squares method. The choosen fitted functions are :

$\operatorname{coef}_{f 1}=A_{1}+B_{1} \cdot \tanh \left[C_{1} \cdot(c n-7)+D_{1}\right]$

$\operatorname{coef}_{f 2}=A_{2}+B_{2} \cdot c n+C_{2} \cdot c n^{2}+D_{2} \cdot c n^{3}$

In these formulas, coef $f_{f 1}$ is related to $a_{1}, b_{1}, c_{1}, d_{1}$ and coef $f_{2}$ represents $a_{2}, b_{2}, c_{2}, d_{2}$ coefficients. The related fitted curves and their equations are shown in Fig. 6 and 7.

Fig. 5, 6 and 7 reveal that the fitted curves and fitted surface are in good accordance with data points. In addition, these regressions curves respect the coefficient of determination citerion where $R^{2} \approx 1$. These proposed fitting functions will be validated in section 4 .

\subsection{Calibration of microscopic failure citerion}

In this section, relationships between macroscopic tensile strength $\sigma_{M}$ and microscopic parameters is studied. Following the same method, series of tensile test simulations with different values of $\left(E_{m}, r_{m}, \sigma_{m}\right.$ and $c n$ ) (Table 3$)$ were carried out in order to determine analytic laws. Here, $4 \times 4 \times 5 \times 10 \times 8=6,400$ simulations were performed.

After initial analyses, as illustrated on Fig. 8(a), one notes that if values of $E_{m}, r_{m}, \sigma_{m}$ and $c n$ are fixed, $\sigma_{M}$ is proportional to $\sigma_{m}$ :

$$
\sigma_{M}=k \times \sigma_{m}
$$



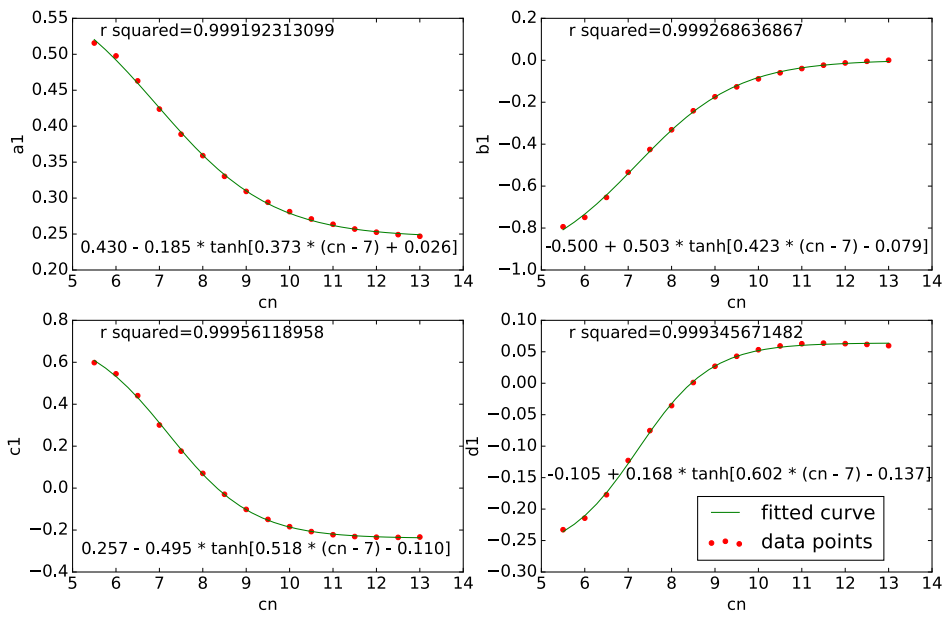

Fig. 6 Fitted curves for $a_{1}, b_{1}, c_{1}$ and $d_{1}$ coefficients of $f_{1}$ versus coordination number $c_{n}$
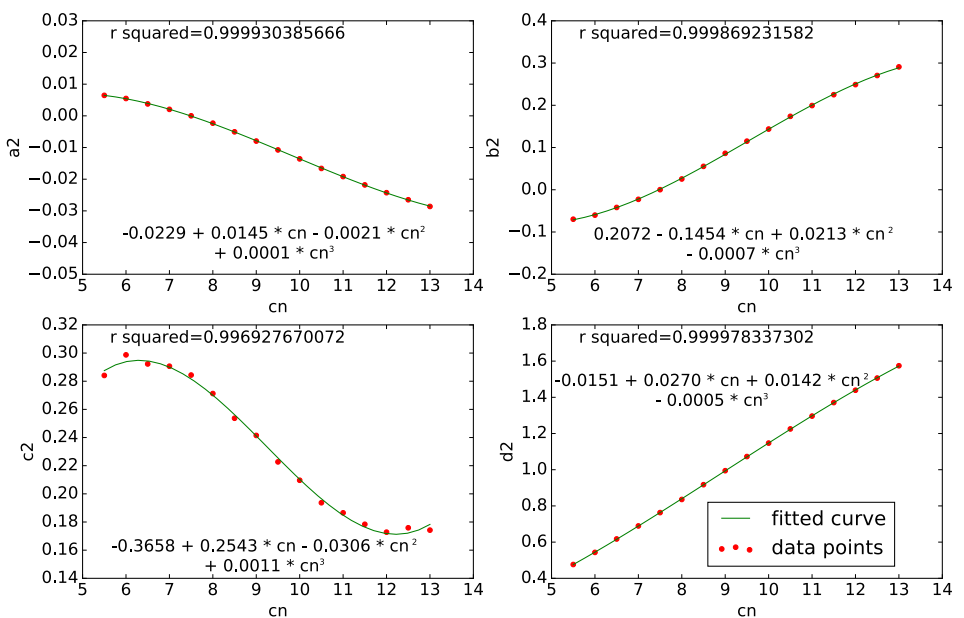

Fig. 7 Fitted curves for $a_{2}, b_{2}, c_{2}$ and $d_{2}$ coefficients of $f_{2}$ versus coordination number $c_{n}$

In addition, Fig. 8(b) reveals that this $k$ ratio does not depend on value of $E_{m}$.

Therefore, a fitting function $k=f_{3}\left(r_{m}, c n\right)$ is expected. After examining many functions, it was found that the relation $k=f_{3}\left(r_{m}, c n\right)$ could be well described by the following relation:

$$
\begin{aligned}
k=f_{3}\left(r_{m}, c n\right)= & {\left[\left(m_{0}+n_{0} \cdot c n+p_{0} \cdot c n^{2}\right)+r_{m} \cdot\left(m_{1}+n_{1} \cdot c n+p_{1} \cdot c n^{2}+q_{1} \cdot c n^{3}\right)\right.} \\
& \left.+r_{m}^{2} \cdot\left(m_{2}+n_{2} \cdot c n+p_{2} \cdot c n^{2}\right)\right]
\end{aligned}
$$

The non-linear least square algorithm gives the following values for the coefficients : 
Table 3 Value of microscopic parameters

\begin{tabular}{ccc}
\hline & Values & Tot. number \\
\hline Sample & 1234 & 4 \\
$E_{m}(\mathrm{GPa})$ & 500100015002000 & 4 \\
$r_{m}(-)$ & 0.20 .40 .60 .81 .0 & 5 \\
$\sigma_{m}(\mathrm{MPa})$ & 2468501003005007001000 & 10 \\
$c n(-)$ & 678910111213 & 8 \\
\hline
\end{tabular}

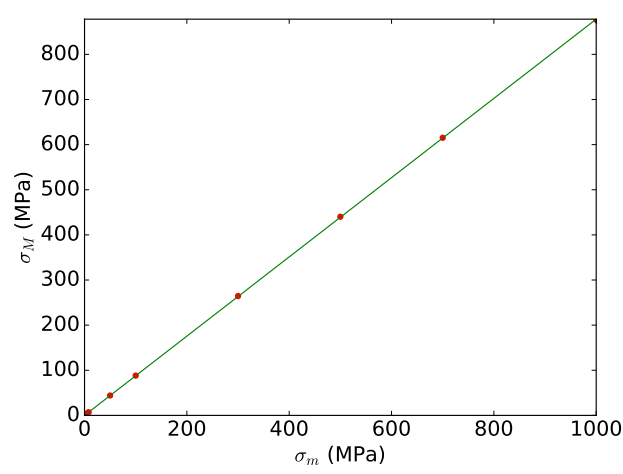

(a) Evolution of $\sigma_{M}$ versus $\sigma_{m}$ when $E_{m}, r_{m}, c n$ are fixed

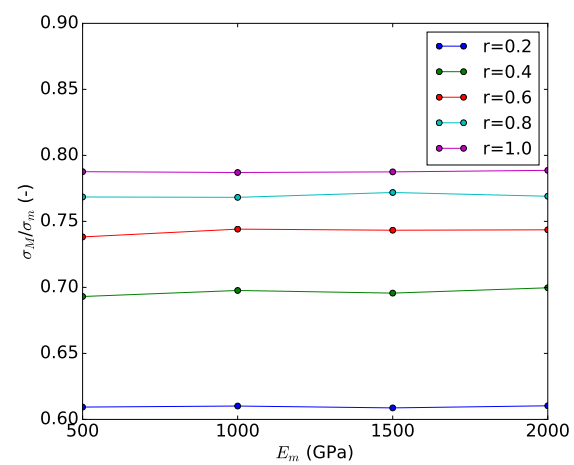

(b) Evoluation of $k$ ratio versus $E_{m}$ (case $c_{n}=7$ )

Fig. 8 Evolution of $\sigma_{M}$ versus microscopic parameters

$\begin{array}{cc}m_{0} & -0.56467 \\ n_{0} & 0.20824 \\ p_{0} & -0.00760 \\ m_{1} & 0.67697 \\ n_{1} & 0.09328 \\ p_{1} & -0.02298 \\ q_{1} & 0.00095 \\ m_{2} & -0.73898 \\ n_{2} & 0.08438 \\ p_{2} & -0.00240\end{array}$

Finally, Fig. 9 reveals that the obtained fitted surface is in good accordance with data points. Nonlinear regression has a good coefficient of determination $\left(R^{2} \approx 1\right)$, indicating an acceptable result of fit. The proposed fitting function will be validate in section 4 .

After analyzing the numerical data, adequate fitting functions which describe correctly the relations between microscopic and macroscopic parameters were found. Thanks to these fitting functions, the value of microscopic parameters could be computed directly from the desired value of macroscopic parameters. In other word, experimental value of mechanical properties of the simulated material could be used directly without fastidious trial-and-error calibration. The proposed calibration method will be validated in the next section through various numerical tests. 


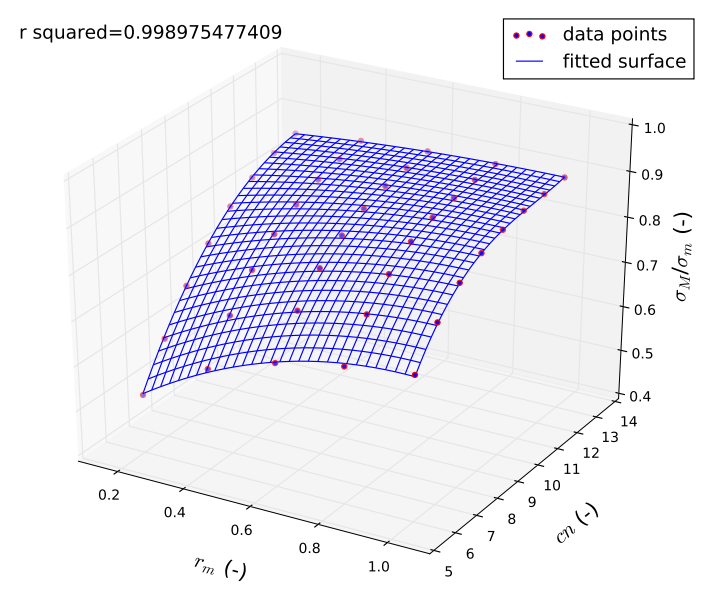

Fig. 9 Fitting results of function $k=f_{3}\left(r_{m}, c n\right)$

\section{Validation of calibration method}

To validate the fitting function proposed in section 3 , the behaviours of two typical brittle materials, i.e. soda-lime glass and alumina, are used as reference. The main mechanical properties of these materials are given in Table 4.

Table 4 Mechanical parameters of reference materials

\begin{tabular}{lcc}
\hline Properties & Glass & Alumina \\
\hline Young's modulus E $(\mathrm{GPa})$ & 72 & 340 \\
Poisson's ratio $v$ & 0.23 & 0.24 \\
Tensile strength $\sigma(\mathrm{MPa})$ & 50 & 380 \\
\hline
\end{tabular}

\subsection{Tensile test simulation}

To validate the proposed calibration method, series of tensile test simulations were carried out. Values of microscopic parameters $\left(r_{m}, E_{m}\right)$ were computed for the two reference materials (see Table 4) by using the given proposed fitting functions (see Equations 17, 18, 19, 20). These values were used as microscopic parameters to perform tensile test simulations. The macroscopic Young's modulus and Poisson's ratio "measured" by these tests were compared to the expected values of the reference materials (see Table 4).

Results are summarized in Tables 5 and 6 . One observes that the maximal difference is lower than $2 \%$, which is an acceptable result. Consequently, the proposed relations between macroscopic parameters and microscopic parameters related to elastic behaviour are validated. 
Table 5 Validation results for glass

\begin{tabular}{ccccccc}
\hline$c n$ & $\begin{array}{c}r_{m} \\
\text { computed }\end{array}$ & $\begin{array}{c}E_{m} \\
\text { computed } \\
(\mathrm{GPa})\end{array}$ & $\begin{array}{c}v_{M} \\
\text { obtained } \\
(-)\end{array}$ & $\begin{array}{c}\text { Difference } \\
(\%)\end{array}$ & $\begin{array}{c}E_{M} \\
\text { obtained } \\
(\mathrm{GPa})\end{array}$ & $\begin{array}{c}\text { Difference } \\
(\%)\end{array}$ \\
\hline 6 & 0.506 & 613.94 & 0.231 & 0.533 & 72.26 & 0.365 \\
7 & 0.461 & 613.52 & 0.230 & 0.103 & 72.06 & 0.085 \\
8 & 0.417 & 636.18 & 0.230 & 0.125 & 71.61 & 0.538 \\
9 & 0.380 & 655.58 & 0.230 & 0.030 & 72.44 & 0.616 \\
10 & 0.348 & 677.94 & 0.230 & 0.167 & 71.74 & 0.364 \\
11 & 0.320 & 705.07 & 0.230 & 0.191 & 71.36 & 0.884 \\
12 & 0.298 & 723.72 & 0.230 & 0.112 & 71.00 & 1.378 \\
13 & 0.283 & 725.14 & 0.230 & 0.163 & 71.22 & 1.077 \\
\hline
\end{tabular}

Table 6 Validation results for alumina

\begin{tabular}{ccccccc}
\hline$c n$ & $\begin{array}{c}r_{m} \\
\text { computed }\end{array}$ & $\begin{array}{c}E_{m} \\
\text { computed } \\
(\mathrm{GPa})\end{array}$ & $\begin{array}{c}v_{M} \\
\text { obtained (-) }\end{array}$ & $\begin{array}{c}\text { Difference } \\
(\%)\end{array}$ & $\begin{array}{c}E_{M} \\
\text { obtained } \\
(\mathrm{GPa})\end{array}$ & $\begin{array}{c}\text { Difference } \\
(\%)\end{array}$ \\
\hline 6 & 0.478 & 3378.29 & 0.241 & 0.490 & 341.37 & 0.404 \\
7 & 0.431 & 3439.53 & 0.240 & 0.000 & 340.29 & 0.085 \\
8 & 0.384 & 3695.23 & 0.240 & 0.000 & 337.98 & 0.593 \\
9 & 0.338 & 4058.51 & 0.240 & 0.000 & 341.88 & 0.554 \\
10 & 0.294 & 4646.18 & 0.241 & 0.324 & 337.70 & 0.677 \\
11 & 0.251 & 5576.21 & 0.241 & 0.353 & 335.66 & 1.276 \\
12 & 0.215 & 6820.35 & 0.240 & 0.000 & 336.62 & 0.995 \\
13 & 0.189 & 8187.01 & 0.239 & 0.404 & 345.80 & 1.705 \\
\hline
\end{tabular}

4.2 Validation through hydrostatic compressive test

In this section, the sensitivity of the proposed calibration method is examined by changing sample shape, sample size and boundary conditions. Series of hydrostatic compression tests were performed. Hydrostatic compression test are commonly involved to determine bulk modulus of materials. To perform simulation, a uniform pressure is imposed on the surface of spherical samples as shown on Fig. 10. Spherical samples have $10 \mathrm{~mm}$ of diameter and contains around 15,000 discrete elements.

The bulk elastic properties of a material determines how much it will compress under a given pressure. The ratio of the imposed pressure to the fractional volume compression is called the bulk modulus $K$ of the material (Equation 23).

$$
K=-\frac{P}{\frac{\Delta V}{V}}
$$

where $V$ is the related sample volume, $\Delta V$ the volume change and $P$ is the imposed pressure.

In the discrete element framework, pressure does not exist. Indeed, the imposed pressure can be computed as:

$$
P=\frac{1}{S} \sum_{j=1}^{N} \mathbf{f}_{\mathbf{j}}=\frac{1}{4 \Pi R^{2}} \sum_{j=1}^{N} \mathbf{f}_{\mathbf{j}}
$$

where $j$ is an element that belongs to the sample surface, $\mathbf{f}_{\mathbf{j}}$ is the imposed force on element $j, S$ is the total surface and $R$ is sample radius. According to the material strength theory, the bulk modulus can be computed through Young's modulus and Poisson's ratio as:

$$
K=\frac{E}{3(1-2 v)}
$$




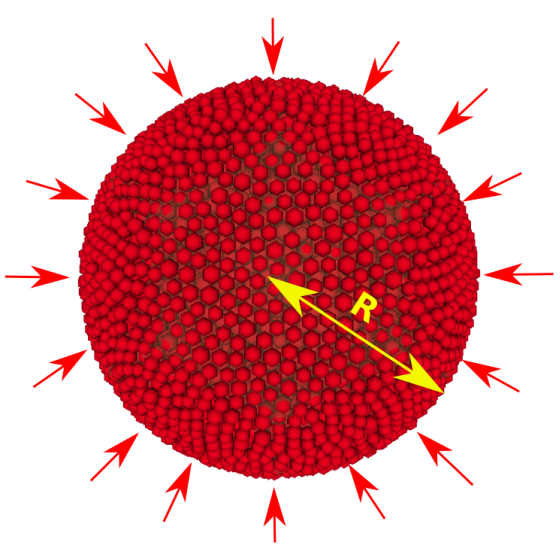

Fig. 10 Configuration of hydrostatic compression test simulation

By using Equation 25 and mechanical properties of glass and alumina (see Table 4), theoretical bulk modulus values of glass and alumina are $44.4 \mathrm{GPa}$ and $218 \mathrm{GPa}$ respectively. Values of measured macroscopic bulk modulus $K_{M}$, using Equations 23 and 24, are compared to these theoretical values.

A typical behaviour resulting from hydrostatic compression test simulations is reported in Fig. 11. The macroscopic bulk modulus $K_{M}$ converges quickly towards a stable value. This stable value is taken as reference for the given hydrostatic test. Results are shown in Table 7 and 8 . These results show that difference between numerical and theoretical values of bulk modulus are lower than $2 \%$ for the two reference materials. Consequently, the proposed relations between macroscopic parameters and microscopic parameters related to elastic behaviour can be validated again through hydrostatic compression test. In other words, the proposed calibration method remains accurate for different type of numerical experiment and for different shape and sizes of sample (dimension and number of discrete elements).

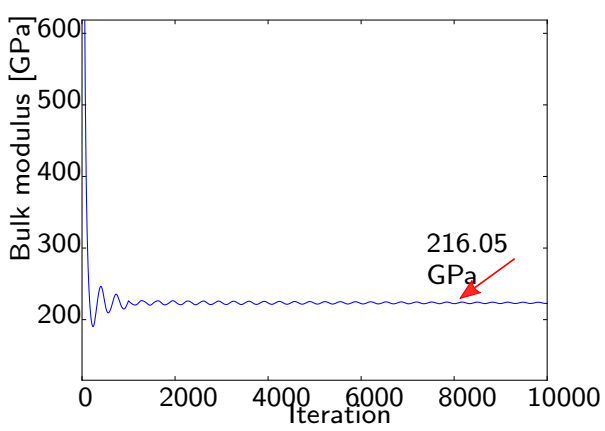

Fig. 11 Evolution of macroscopic bulk modulus $K_{M}$ during a hydrostatic compression test simulation for alumina, case of $c n=10$ 
Table 7 Validation results through hydrostatic compression test for glass

\begin{tabular}{ccccc}
\hline$c n$ & $\begin{array}{c}r_{m} \\
(-)\end{array}$ & $\begin{array}{c}E_{m} \\
(\mathrm{GPa})\end{array}$ & $\begin{array}{c}K_{M} \\
\text { mesured } \\
(\mathrm{GPa})\end{array}$ & $\begin{array}{c}\text { Difference } \\
(\%)\end{array}$ \\
\hline 6 & 0.506 & 613.94 & 44.319 & -0.282 \\
7 & 0.461 & 613.52 & 44.555 & 0.249 \\
8 & 0.417 & 636.18 & 44.428 & -0.036 \\
9 & 0.380 & 655.58 & 44.564 & 0.269 \\
10 & 0.348 & 677.94 & 44.566 & 0.274 \\
11 & 0.320 & 705.07 & 44.320 & -0.280 \\
12 & 0.298 & 723.72 & 44.201 & -0.548 \\
13 & 0.283 & 725.14 & 44.307 & -0.310 \\
\hline
\end{tabular}

Table 8 Validation results through hydrostatic compression test for alumina

\begin{tabular}{ccccc}
\hline$c n$ & $r_{m}$ & $\begin{array}{c}E_{m} \\
(-)\end{array}$ & $\begin{array}{c}K_{M} \\
\text { mesured } \\
(\mathrm{GPa})\end{array}$ & $\begin{array}{c}\text { Difference } \\
(\%)\end{array}$ \\
\hline 6 & 0.478 & 3378.29 & 215.312 & -1.210 \\
7 & 0.431 & 3439.53 & 216.325 & -0.745 \\
8 & 0.384 & 3695.23 & 215.526 & -1.112 \\
9 & 0.338 & 4058.51 & 216.146 & -0.827 \\
10 & 0.294 & 4646.18 & 216.054 & -0.869 \\
11 & 0.251 & 5576.21 & 214.793 & -1.448 \\
12 & 0.215 & 6820.35 & 215.718 & -1.024 \\
13 & 0.189 & 8187.01 & 221.124 & 1.457 \\
\hline
\end{tabular}

\subsection{Validation through Brazilian test}

To validate the proposed relation between macroscopic tensile strength $\sigma_{M}$ and microscopic parameters, series of Brazilian test simulations were carried out. Brazilian tests are commonly used for characterization of brittle materials such as ceramic, concrete, refractory. Brazilian tests are performed by applying a vertical compressive load across the diameter of a disk sample. In the rupture phase, a crack appears along the vertical diameter of the disk, due to tensile stresses induced horizontally by the geometry of sample [5].

Again, validation process is given for glass and alumina materials. For each value of coordination number, the corresponding value of $r_{m}$ obtained in the previous section (Table 5 and 6) was used to compute microscopic fracture threshold $\sigma_{m}$ value by using the Equation 22. This value of $\sigma_{m}$ was used to perform Brazilian test simulations. The virtual disk samples have $50 \mathrm{~mm}, 10 \mathrm{~mm}$ of thickness and contain around 20,000 discrete elements. According to experimental and numerical studies in literature, crack initiation at the center of the disk sample is considered to be crucial for the test validity [9,11,48]. C. Fairhurst, in [11], stated that "failure may occur away from the center of the disk for small angles of loading contact area with material of low compression tension ratios. In such cases, the tensile strength as usually calculated from test results, is lower than the true value". Conforming to this observation, opposite vertical displacements are imposed on two circular $\operatorname{arcs}$ of $2 \alpha=24^{\circ}$ in this study as shown on Figure 12.

Vertical forces at upper and lower loading contact area, $P_{\text {up }}$ and $P_{\text {down }}$ respectively, resulting from the imposed displacement are measured during simulations. The applied force $P$ is computed as:

$$
P=\frac{\left|P_{\mathrm{up}}\right|+\left|P_{\mathrm{down}}\right|}{2}
$$




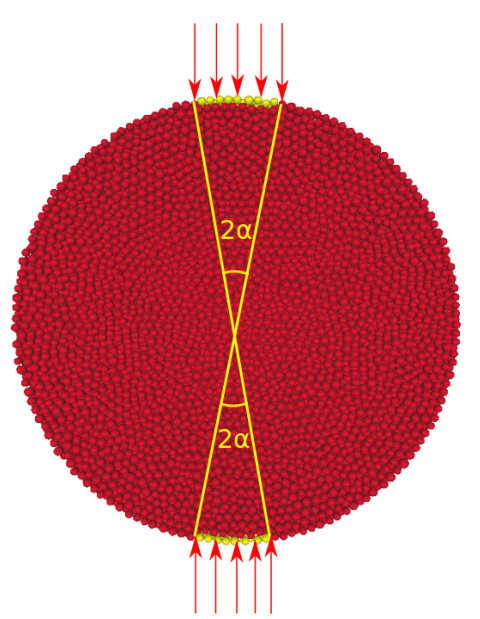

Fig. 12 Configuration of the virtual Brazilian test

According to literature [48], the tensile strength can be computed as:

$$
\sigma_{M}=\frac{2 \cdot P_{c}}{\pi \cdot t \cdot D}
$$

where $P_{c}$ is the critical load (it is also the maximum applied load during the test), $t$ and $D$ are the disk thickness and the disk diameter respectively.

The typical behaviours resulting from the Brazilian test simulation are reported in Fig. 13, 14, and 15. In Fig. 13, a sudden decreasing of the average force $P$ indicates the failure of the virtual sample. The corresponding value of $P$ is considered as the critical load in order to compute the macroscopic tensile strength $\sigma_{M}$ of the sample by using Equation 27. Fig. 14 shows that the crack is initiated near the center of the disk and propagate along vertical diameter, as expected. In conclusion, the crack initiation and propagation are relevant with theoretical predictions and experimental observations [9].

In order to highlight the interest of the virial stress computation, as described in section 2.2, the evolution of stress along the diameter is monitored. Fig. 15 shows both the theoretical horizontal stress evolution and the numerical one. This comparison was performed before the failure of sample, when the applied force $P \approx 36.48 \mathrm{kN}$ (Fig. 13). The theoretical stress distribution on the loading diameter is given by the following relationship [18] [38]:

$$
\sigma=\frac{2 p}{\pi}\left\{\frac{\sin 2 \alpha}{1-2 \rho^{2} \cos 2 \alpha+\rho^{4}}\left[1-\rho^{2}\right]-\arctan \left[\frac{\rho^{2} \sin 2 \alpha}{1-\rho^{2} \cos 2 \alpha}\right]-\alpha\right\}
$$

where :

- $\sigma$ is the horizontal normal stress,

- $p$ is the load per unit area,

- $t$ is the disk thickness,

- $R$ is the disk radius of the disk,

- $r$ is distance from a point in disk to the center,

- $\rho$ is equal to $r / R$ and

- $\alpha$ is the half central angle of the applied distributed load. 
Note that, in Fig. 15, the numerical blue curve is given thanks to the Gaussian Kernel interpolation method available in the Paraview ${ }^{2}$ software. This figure reveals a good quantitative accordance between theoretical curve (continuous dotted curve) and numerical curve, although there are discrepancies towards the loading areas. The possible reason for these discrepancies may come from the edge effect that influence on the virial stress computation at the boundaries. In fact, at the boundaries of domain, discrete elements are cut by domain boundaries, then their coordination numbers are one half of the coordination numbers of the internal elements. This could raise errors in the computation.

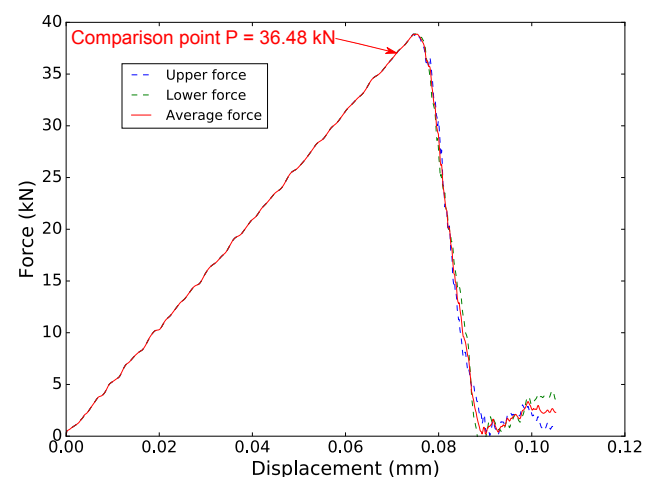

Fig. 13 Force - displacement curve during Brazilian test, case $c n=10$, glass
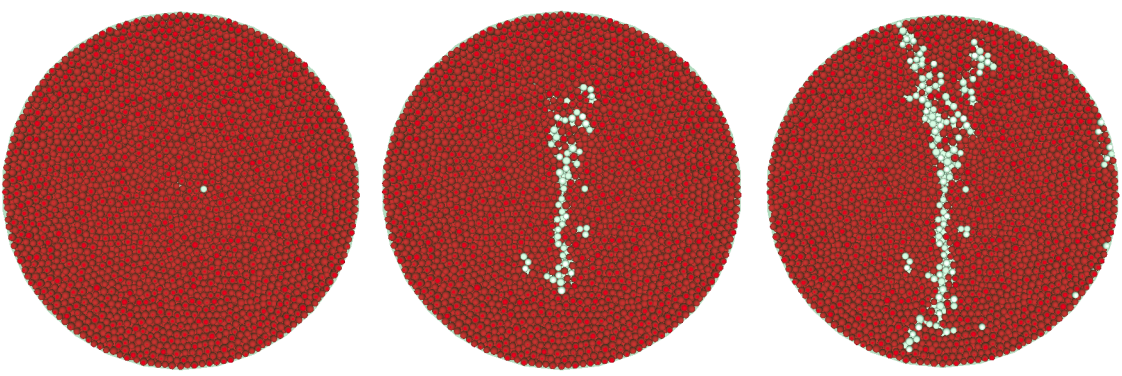

Fig. 14 Crack propagation of Brazilian test

The macroscopic tensile strength $\sigma_{M}$ obtained by Brazilian tests were compared to the expected theoritical values of the tensile strength of glass and alumina (50 MPa and 380 MPa, respectively, see Table 4). Synthesis of the virtual Brazil test with the proposed fracture criterion (see section 2.2) is shown in the Table 9 and 10. Quantitatively, differences are quite acceptable for the two reference materials. In addition, crack initiation and propagation and stress distribution obtained by DEM simulation are compatible with material strength theory and experimental observations. Consequently, the proposed relation between $\sigma_{M}$ and microscopic parameters of DEM could be validated. Furthermore, the proposed fracture

2 see www.paraview.org 


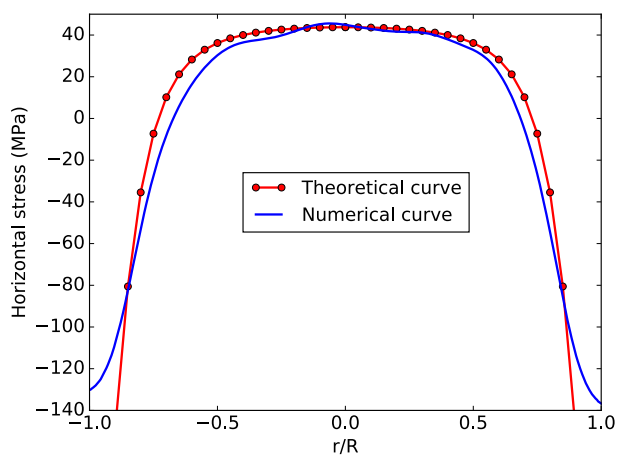

Fig. 15 Horizontal stress distribution along vertical diameter of sample, case $c n=10$, glass, when applied force $P=36.48 \mathrm{kN}$ (see Fig. 13)

Table 9 Validation of brittle behaviour calibration for glass

\begin{tabular}{ccccc}
\hline$c n$ & $r_{m}$ & $\sigma_{m}(\mathrm{MPa})$ & $\sigma_{M}(\mathrm{MPa})$ & Difference $(\%)$ \\
\hline 6 & 0.506 & 78.08 & 50.94 & 1.88 \\
\hline 7 & 0.461 & 70.58 & 49.59 & 1.80 \\
\hline 8 & 0.417 & 65.79 & 49.13 & 1.74 \\
\hline 9 & 0.380 & 62.43 & 49.74 & 2.20 \\
\hline 10 & 0.348 & 60.08 & 50.27 & 2.05 \\
\hline 11 & 0.320 & 58.44 & 52.08 & 4.16 \\
\hline 12 & 0.298 & 57.37 & 53.08 & 6.15 \\
\hline 13 & 0.283 & 56.80 & 53.49 & 6.99
\end{tabular}

Table 10 Validation of brittle behaviour calibration for alumina

\begin{tabular}{ccccc}
\hline$c n$ & $r_{m}$ & $\sigma_{m}(\mathrm{MPa})$ & $\sigma_{M}(\mathrm{MPa})$ & Difference (\%) \\
\hline 6 & 0.478 & 601.22 & 377.30 & 0.97 \\
\hline 7 & 0.431 & 543.05 & 363.01 & 4.47 \\
\hline 8 & 0.384 & 505.90 & 359.54 & 5.38 \\
\hline 9 & 0.338 & 480.10 & 369.24 & 2.83 \\
\hline 10 & 0.294 & 461.84 & 368.16 & 3.12 \\
\hline 11 & 0.251 & 448.82 & 385.09 & 2.00 \\
\hline 12 & 0.215 & 439.89 & 396.17 & 4.26 \\
\hline 13 & 0.189 & 435.00 & 406.88 & 7.08 \\
\hline
\end{tabular}

criterion and the virial stress computation seem to be able to simulate fracture phenomena of brittle materials under complex loadings. Indeed, this validation involves only (indirect) tensile test.

4.4 Brittle behaviour through brittle torsion test

In this section, the proposed calibration method is applied for brittle torsion test simulation. Fig. 16 presents the geometric model used to simulate the quasi-static torsion test. These virtual cylindrical samples have diameter of $4 \mathrm{~mm}$, length of $100 \mathrm{~mm}$ and contain around 10,000 discrete elements. The samples are subjected to progressive rotations $\phi_{x}$ and $-\phi_{x}$ about the $\mathbf{X}$ axis on the $x$ Min and $x$ Max opposite faces. The coordination number is arbitrary 


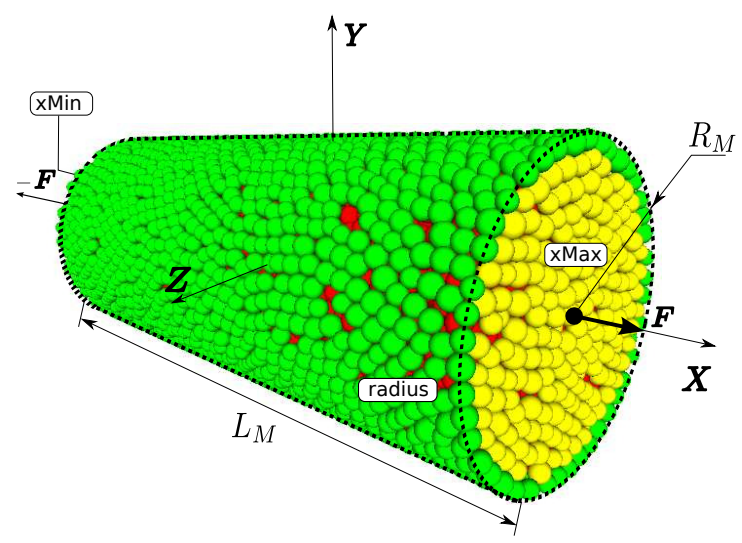

Fig. 16 Discrete domain for torsion tests

set at 10 and the corresponding values of microscopic parameters $\left(r_{m}, E_{m}\right.$ and $\left.\sigma_{m}\right)$ are used to perform simulations of both reference materials.

To compute the macroscopic fracture stress, the forces $\mathbf{F}_{\mathbf{p}}$ and torques $\mathbf{M}_{\mathbf{p}}$ applied on opposite faces are monitored. The macroscopic torsion torque $M_{M x}$ can be obtained from these quantities as follows:

$$
\begin{aligned}
M_{M x}^{x M i n} & =\sum_{p=1}^{N_{x M i n}}\left(\mathbf{M}_{\mathbf{p}}+\mathbf{O}_{\mathbf{1}} \mathbf{G}_{\mathbf{p}} \wedge \mathbf{F}_{\mathbf{p}}\right) \cdot \mathbf{X} \\
M_{M x}^{x M a x} & =\sum_{p=1}^{N_{x M a x}}\left(\mathbf{M}_{\mathbf{p}}+\mathbf{O}_{\mathbf{2}} \mathbf{G}_{\mathbf{p}} \wedge \mathbf{F}_{\mathbf{p}}\right) \cdot \mathbf{X}
\end{aligned}
$$

where the points $O_{1}$ and $O_{2}$ are the centers of $x$ Min and $x M a x$ faces and $G_{P}$ is the center of a discrete element $p$. Then, the macroscopic torsion torque $M_{M x}$ is taken as the mean of $M_{M x}^{x M i n}$ and $M_{M x}^{x M a x}$. Based on the material strength theory, the maximal macroscopic shear stress can be obtained as:

$$
\tau_{M_{\max }}=\frac{M_{M x}}{I o} R_{M}
$$

where $R_{M}$ is the radius of the discrete domain and $I o$ is the polar moment of inertia which is defined as:

$$
I o=\frac{\pi R_{M}^{4}}{2}
$$

Using the Rankine criterion, the macroscopic tensile strength which is the maximal macroscopic principal stress can be expressed as:

$$
\sigma_{M}=\left(\tau_{M_{\max }}\right)_{\text {fracture }}
$$

where $\left(\tau_{M_{\max }}\right)_{\text {fracture }}$ is the maximal macroscopic shear stress measured at the fracture of the numerical sample. The values of macroscopic tensile strength obtained by torsion test are compared with targeted values of tensile strength for reference materials. A typical behaviour resulting from torsion test is reported in Fig. 17. In addition, Table 11 summarizes the simulation results. The difference between obtained values of $\sigma_{M}$ and targeted values are quite acceptable of two materials. Moreover, at the structure scale, the crack geometry 


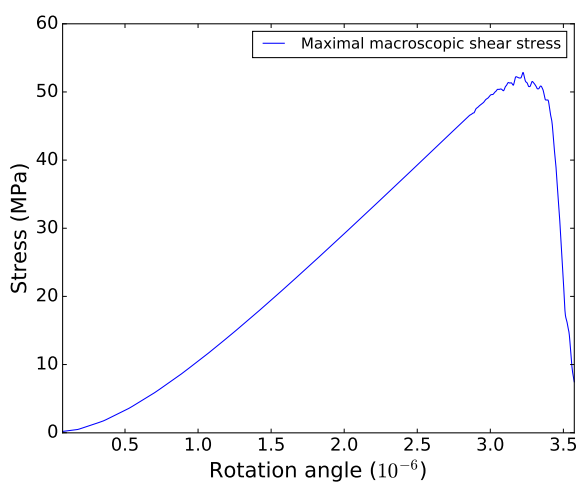

Fig. 17 Shear stress curve during torsion test for glass

Table 11 Torsion simulation results

\begin{tabular}{|c||cc|cc|c|}
\hline & $r_{m}$ & $\sigma_{m}(\mathrm{MPa})$ & $\sigma_{M}(\mathrm{MPa})$ & $\sigma(\mathrm{MPa})$ & Difference $(\%)$ \\
\hline Glass & 0.348 & -60.8 & 53.04 & 53.04 & 6 \\
\hline Alumina & 0.294 & -461.4 & 394.23 & 394.23 & 3.69 \\
\hline
\end{tabular}

seems in agreement with the brittle fracture theory (fig. 18) that predicts an helical crack surface oriented at $45^{\circ}$ to the main axis of the numerical sample. Consequently, the proposed relation between $\sigma_{M}$ and microscopic parameter of DEM could be validated when brittle failure occurs in shear experiments.
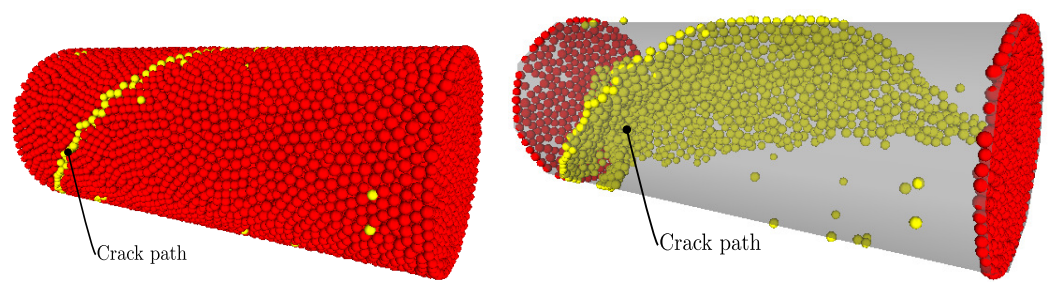

Fig. 18 View of crack path in a torsional test; the discrete elements in which the fracture criterion is fulfilled are highlighted (View showing all discrete elements and view showing only critical discrete elements)

\subsection{Brittle behaviour through compression test}

In this section, the proposed calibration method is applied for compressive tests. These tests are quite similar to tensile tests previously described in section 2.4. Again, silica glass and alumina are used as reference materials. Here, the difference consists in adding a negative failure criterion in compression arbitrary fixed to ten times higher than the tensile strength, i.e, $\sigma=-500 \mathrm{MPa}$ for silica glass and $\sigma=-3,800 \mathrm{MPa}$ for alumina. Table 12 shows that differences between targeted results and numerical results are less than $2 \%$. These tests are very preliminary and further investigations are planned for validating these results through experimental observations. Indeed, these preliminary results highlights the potentialities of 
Table 12 Compressive simulation results

\begin{tabular}{|c||cc|cc|c|}
\hline & $r_{m}$ & $\sigma_{m}(\mathrm{MPa})$ & $\sigma_{M}(\mathrm{MPa})$ & $\sigma(\mathrm{MPa})$ & Difference $(\%)$ \\
\hline Glass & 0.348 & -600.8 & -501.0 & -500 & 0.2 \\
\hline Alumina & 0.294 & -4618.4 & $-3,843.1$ & $-3,800$ & 1.1 \\
\hline
\end{tabular}

this method. Using 3D quantitative stress tensors enables the development of sophisticated failure criterion such as asymmetric failure strength or Mohr-Coulomb criterion widely used in mechanics of quasi-brittle material such rocks, concretes or refractories.

\section{Conclusion}

The present paper deals with the development of an analytic laws to describe the relationships between microscopic and macroscopic parameters for cohesive beam model in DEM. The proposed analytic laws allow a direct computation of microscopic parameters from desired values of macroscopic parameters, i.e, material parameters. For future DEM simulations, experimental values of mechanical properties of simulated material could be used directly to perform DEM simulation without fastidious trial-and-error calibration which is extremely time consuming.

The proposed analytic laws were validated with different shapes/size of discrete domain, various boundary conditions and numerical experiments. The values of macroscopic parameters obtained by the validation tests are in good accordance with targeted values of materials. Therefore, both accuracy and sensitivity of proposed analytical laws are validated. In addition, the validation by Brazilian test reveals capability of virial stress computation to simulate precisely fracture phenomena of brittle material under complex loading. The crack initiation and propagation simulated by DEM are compatible with theoretical prediction and experimental observations. The proposed fracture criterion may be applied to a large class of brittle materials: geo-materials, civil engineering materials and ceramics.

\section{Acknowledgements}

This study was conducted within the framework of FIRE (Federation of International Refractory Research and Education), project Delta. Financial support from the Nouvelle-Aquitaine region (France) is gratefully acknowledged.

\section{Compliance with ethical standards}

Funding: This study was funded by Nouvelle-Aquitaine region (France).

Conflict of interest The authors declare that they have no conflict of interest.

\section{References}

1. Damien André, Ivan Iordanoff, Jean luc Charles, and Jérome Néauport. Discrete element method to simulate continuous material by using the cohesive beam model. Computer Methods in Applied Mechanics and Engineering, 213216(0):113 - 125, 2012. 
2. Damien André, Bertrand Levraut, Nicolas Tessier-Doyen, and Marc Huger. A discrete element thermomechanical modelling of diffuse damage induced by thermal expansion mismatch of two-phase materials. Computer Methods in Applied Mechanics and Engineering, 318:898-916, 2017.

3. D. Andr, J-L. Charles, and I. Iordanoff. 3D Discrete Element Workbench for Highly Dynamic Thermomechanical Analysis: GranOO. Wiley, 2015.

4. Damien Andr, Mohamed Jebahi, Ivan Iordanoff, Jean luc Charles, and Jrme Nauport. Using the discrete element method to simulate brittle fracture in the indentation of a silica glass with a blunt indenter. Computer Methods in Applied Mechanics and Engineering, 265(0):136 - 147, 2013.

5. Y Belrhiti, JC Dupre, O Pop, A Germaneau, P Doumalin, M Huger, and T Chotard. Combination of brazilian test and digital image correlation for mechanical characterization of refractory materials. Journal of the European Ceramic Society, 37(5):2285-2293, 2017.

6. H. A. Carmona, F. K. Wittel, F. Kun, and H. J. Herrmann. Fragmentation processes in impact of spheres. Physical Review, 77(5):051302, 2008.

7. G. A. D'Addetta, F. Kun, and E. Ramm. On the application of a discrete model to the fracture process of cohesive granular materials. Granular Matter, 4(2):77-90, 2002.

8. G. A. D'Addetta and E. Ramm. A microstructure-based simulation environment on the basis of an interface enhanced particle model. Granular Matter, 8(3):159-174, 2006.

9. Nazife Erarslan and David John Williams. Experimental, numerical and analytical studies on tensile strength of rocks. International Journal of Rock Mechanics and Mining Sciences, 49:21 - 30, 2012.

10. Danilo Estay, Felipe Chacana, Jaime Ibarra, Luis Pérez, and Sheila Lascano. Bond calibration method for youngs modulus determination in the discrete element method framework. Granular Matter, 19(3):60, 2017.

11. C. Fairhurst. On the validity of the brazilian test for brittle materials. International Journal of Rock Mechanics and Mining Sciences \& Geomechanics Abstracts, 1(4):535 - 546, 1964.

12. Ali Fakhimi and Tomas Villegas. Application of dimensional analysis in calibration of a discrete element model for rock deformation and fracture. Rock Mechanics and Rock Engineering, 40(2):193, 2007.

13. Keishi Gotoh and J. L. Finney. Statistical geometrical approach to random packing density of equal spheres. Nature, 252:202-205, 1974.

14. Hamza Haddad, W Leclerc, M Guessasma, C Pélegris, N Ferguen, and E Bellenger. Application of dem to predict the elastic behavior of particulate composite materials. Granular Matter, 17(4):459-473, 2015.

15. Zoltán Halász, Akio Nakahara, So Kitsunezaki, and Ferenc Kun. Effect of disorder on shrinkage-induced fragmentation of a thin brittle layer. Physical Review E, 96(3):033006, 2017.

16. Zhenyu Han, Dion Weatherley, and Ruslan Puscasu. A relationship between tensile strength and loading stress governing the onset of mode $\mathrm{i}$ crack propagation obtained via numerical investigations using a bonded particle model. International Journal for Numerical and Analytical Methods in Geomechanics, 41(18):1979-1991, 2017.

17. H. J. Herrmann. Proceedings of the nato advanced study institute, cargèse, corsica, france, 18-31 july, 1988. In Random Fluctuations and Pattern Growth: Experiments and Models Random Fluctuations and Pattern Growth: Experiments and Models, volume 157 of NATO Science Series E, pages 149-160. Stanley, Harry Eugene; Ostrowsky, N., 1988.

18. G Hondros. The evaluation of poisson's ratio and the modulus of materials of low tensile resistance by the brazilian (indirect tensile) test with particular reference to concrete. Australian J. Appl. Sci., 10(3):243-268, 1959.

19. Haiying Huang. Discrete element modeling of tool-rock interaction. University of Minnesota, 1999.

20. Cédric Hubert, Damien André, L Dubar, I Iordanoff, and JL Charles. Simulation of continuum electrical conduction and joule heating using dem domains. International Journal for Numerical Methods in Engineering, 110(9):862-877, 2017.

21. A. Ibrahimbegovic and A. Delaplace. Microscale and mesoscale discrete models for dynamic fracture of structures built of brittle material. Computers \& Structures, 81(21):1255-1265, 2003.

22. Itasca Consulting Group Inc. Particle flow code in 2 dimensions. 2012.

23. M. Jebahi, D. Andr, I. Terreros, and I. Iordanoff. Discrete Element Method to Model 3D Continuous Materials. Wiley, 2015.

24. Mohamed Jebahi, Frédéric Dau, Ivan Iordanoff, and Jean-Pierre Guin. Virial stress-based model to simulate the silica glass densification with the discrete element method. International Journal for Numerical Methods in Engineering, 112(13):1909-1925, 2017.

25. Jan Kozicki and Frederic V Donze. A new open-source software developed for numerical simulations using discrete modeling methods. Computer Methods in Applied Mechanics and Engineering, 197(4950):4429-4443, 2008.

26. F. Kun and H. J. Herrmann. A study of fragmentation processes using a discrete element method. Computer Methods in Applied Mechanics and Engineering, 138(1-4):3-18, 1996. 
27. HL Langhaar. Dimensional analysis and theory of models, john wiley \& sons. Inc., New York, 1951.

28. Kenneth Levenberg. A method for the solution of certain non-linear problems in least squares. Quarterly of applied mathematics, 2(2):164-168, 1944.

29. G Lilliu and J.G.M van Mier. 3d lattice type fracture model for concrete. Engineering Fracture Mechanics, 70(7):927 - 941, 2003.

30. Laurent Mahéo, Vincent Grolleau, and Gérard Rio. Damping efficiency of the tchamwa-wielgosz explicit dissipative scheme under instantaneous loading conditions. Comptes Rendus Mécanique, 337(1112):722-732, 2009.

31. Michele Marigo and Edmund Hugh Stitt. Discrete element method (dem) for industrial applications: Comments on calibration and validation for the modelling of cylindrical pellets. KONA Powder and Particle Journal, 32:236-252, 2015.

32. Jorge J Moré. The levenberg-marquardt algorithm: implementation and theory. In Numerical analysis, pages 105-116. Springer, 1978.

33. T. Pöschel and S. Luding. Granular Gases, volume Vol. 564 of Lecture Notes in Physics. Pöschel, Thorsten; Luding, Stefan, 2001.

34. T. Pöschel and T. Schwager. Computational granular dynamics. Springer, 2005.

35. D.O. Potyondy and P. A. Cundall. A bonded-particle model for rock. International Journal of Rock Mechanics and Mining Sciences, 41(8):1329-1364, 2004. Rock Mechanics Results from the Underground Research Laboratory, Canada.

36. Jerzy Rojek, Eugenio Onate, Carlos Labra, and Hubert Kargl. Discrete element simulation of rock cutting. International Journal of Rock Mechanics and Mining Sciences, 48(6):996-1010, 2011.

37. E. Rougier, A. Munjiza, and N. W. M. John. Numerical comparison of some explicit time integration schemes used in dem, fem/dem and molecular dynamics. International Journal for Numerical Methods in Engineering, 61(6):856-879, 2004.

38. E Sarris, Z Agioutantis, K Kaklis, and SK Kourkoulis. Numerical simulation of the cracked brazilian disc under diametral compression. In Bifurcations, Instabilities, Degradation in Geomechanics, pages 403-430. Springer, 2007.

39. E. Schlangen and E. J. Garboczi. New method for simulating fracture using an elastically uniform random geometry lattice. International Journal of Engineering Science, 34(10):1131-1144, 1996.

40. E. Schlangen and J. G. M. van Mier. Experimental and numerical analysis of micromechanisms of fracture of cement-based composites. Cement and Concrete Composites, 14(2):105-118, 1992. Special Issue on Micromechanics of Failure in Cementitious Composites.

41. E. Schlangen and J. G. M. van Mier. Simple lattice model for numerical simulation of fracture of concrete materials and structures. Materials and Structures, 25(9):534-542, 1992.

42. Wenjie Shiu, Frederic Victor Donze, and Laurent Daudeville. Discrete element modelling of missile impacts on a reinforced concrete target. International Journal of Computer Applications in Technology, 34(1):33-41, 2009.

43. Gilbert Strang. Linear algebra and its applications. Harcourt, Brace, Jovanovich, Publishers, 3rd ed edition, 1988.

44. F. A. Tavarez and M. E. Plesha. Discrete element method for modelling solid and particulate materials. International Journal for Numerical Methods in Engineering, 70:379-404, 2007.

45. I. Terreros, I. Iordanoff, and J.L. Charles. Simulation of continuum heat conduction using dem domains. Computational Materials Science, 69(0):46 - 52, 2013.

46. S. P. Timoshenko. History of strength of materials: with a brief account of the history of theory of elasticity and theory of structures. Dover, New York, NY.

47. Min Wang and Ping Cao. Calibrating the micromechanical parameters of the pfc2d (3d) models using the improved simulated annealing algorithm. Mathematical Problems in Engineering, 2017, 2017.

48. QZ Wang, XM Jia, SQ Kou, ZX Zhang, and P-A Lindqvist. The flattened brazilian disc specimen used for testing elastic modulus, tensile strength and fracture toughness of brittle rocks: analytical and numerical results. International Journal of Rock Mechanics and Mining Sciences, 41(2):245-253, 2004.

49. Budong Yang, Yue Jiao, and Shuting Lei. A study on the effects of microparameters on macroproperties for specimens created by bonded particles. Engineering Computations, 23(6):607-631, 2006.

50. Gao-Feng Zhao, Qiuyue Yin, Adrian R. Russell, Yingchun Li, Wei Wu, and Qin Li. On the linear elastic responses of the $2 \mathrm{~d}$ bonded discrete element model. International Journal for Numerical and Analytical Methods in Geomechanics, 0(0).

51. Min Zhou. A new look at the atomic level virial stress: on continuum-molecular system equivalence. Proceedings of the Royal Society of London. Series A: Mathematical, Physical and Engineering Sciences, 459(2037):2347-2392, September 2003. 